\title{
Radiation-Induced Magnetoresistance Oscillations in a 2D Electron Gas
}

\author{
Adam C. Durst, Subir Sachdev, N. Read, and S. M. Girvin \\ Department of Physics, Yale University, P.O. Box 208120, New Haven, CT 06520-8120
}

(Dated: January 29, 2003)

\begin{abstract}
Recent measurements of a $2 \mathrm{D}$ electron gas subjected to microwave radiation reveal a magnetoresistance with an oscillatory dependence on the ratio of radiation frequency to cyclotron frequency. We perform a diagrammatic calculation and find radiation-induced resistivity oscillations with the correct period and phase. Results are explained via a simple picture of current induced by photo-excited disorder-scattered electrons. The oscillations increase with radiation intensity, easily exceeding the dark resistivity and resulting in negative-resistivity minima. At high intensity, we identify additional features, likely due to multi-photon processes, which have yet to be observed experimentally.
\end{abstract}

PACS numbers: 73.40.-c, 78.67.-n, 73.43.-f

The electrical transport properties of a $2 \mathrm{D}$ electron gas (2DEG) in a perpendicular magnetic field have been studied extensively over the past two decades in connection with the quantum Hall effects. However, recent experiments, in which such systems are subjected to electromagnetic radiation, reveal a surprising new phenomenon. The initial experiments of Zudov et al. [1, as well as more detailed subsequent studies conducted by Mani et al. [2] and Zudov et al. [3], show that a peculiar oscillation of the longitudinal resistance is induced by the presence of microwave radiation in systems at high filling factor. Unlike the familiar Shubnikov-de Haas $(\mathrm{SdH})$ oscillations which are controlled by the ratio of the chemical potential, $\mu$, to the cyclotron frequency, $\omega_{c}$, these radiation-induced oscillations are controlled by the ratio of the radiation frequency, $\omega$, to the cyclotron frequency. According to Ref. 2, the minimum resistance values are obtained near $\omega / \omega_{c}=$ integer $+1 / 4$. These results are surprising since naively one would only expect a peak at $\omega=\omega_{c}$ due to heating at the cyclotron resonance. Interest in this phenomenon was heightened when, using high mobility samples, both Mani et al. 2] and Zudov et al. [3] observed that as the radiation intensity is increased, the minimum resistance values approach zero and give rise to new zero-resistance states. In this Letter, we present calculations which reveal the origin of the resistance oscillations, explain their period and phase, hint at the manner in which zero-resistance states may develop, and predict higher-order effects, likely due to multi-photon processes, which set in at high intensity.

It is instructive to begin by exploring a crude treatment through which a simple physical picture can be established. The electronic states of $2 \mathrm{D}$ electrons without disorder in a perpendicular magnetic field, $B$, are the Landau levels, $\epsilon_{n}=n \omega_{c}$, where $\omega_{c}=e B / m^{*} c$, the $n$ are nonnegative integers, and $m^{*}$ is the effective mass. (Here, and always, we set $\hbar=1$ and drop the constant energy shift of $\omega_{c} / 2$.) Each level has a degeneracy per unit area of $1 / 2 \pi \ell^{2}$ where $\ell=\sqrt{c / e B}$ is the magnetic length. In the presence of disorder, the Landau levels are broadened. In correspondence with experiment, we

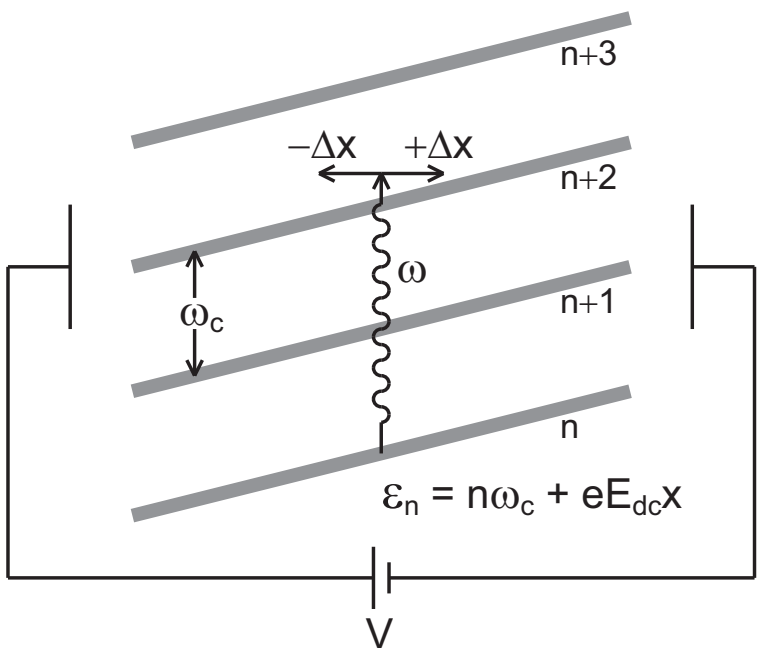

FIG. 1: Simple picture of radiation-induced disorder-assisted current. Landau levels are tilted by the applied dc bias. Electrons absorb photons and are excited by energy $\omega$. Photoexcited electrons are scattered by disorder and kicked to the right or to the left by a distance $\pm \Delta x$. If the final density of states to the left exceeds that to the right, dc current is enhanced. If vice versa, dc current is diminished. Note that electrons initially near the center of a Landau level (where the initial density of states is greatest) will tend to flow uphill for $\omega / \omega_{c} \approx$ integer $+1 / 4$.

consider states occupied up to high Landau levels (the filling factor is about 50 for the experiments of Ref. 2). We suppose that the normalized eigenstates in the presence of disorder are $\psi_{\alpha}(\mathbf{r})$, with eigenvalues $\epsilon_{\alpha}$. Now we turn on the microwave radiation. When an electron absorbs a photon, it is excited by an energy $\omega$. In the absence of disorder, conductivity is independent of the distribution of electrons over Landau levels (Kohn's theorem [4]) so photo-excited electrons can make no additional contribution to the dc current. In the presence of disorder, these electrons can be scattered by impurities as they are excited. We use a generalization of Fermi's golden rule to obtain a position-dependent rate. To second order in the radiation, the rate at which an elec- 
tron is scattered from initial state $\alpha^{\prime}$ to final position $\mathbf{r}$ is $w_{\alpha^{\prime}}(\mathbf{r})=2 \pi \sum_{\alpha}\left|\psi_{\alpha}(\mathbf{r})\right|^{2} \delta\left(\epsilon_{\alpha}-\epsilon_{\alpha^{\prime}}-\omega\right)\left|\left\langle\alpha|e E \hat{x}| \alpha^{\prime}\right\rangle\right|^{2}$, where $\left\langle\alpha|e E \hat{x}| \alpha^{\prime}\right\rangle$ is the matrix element of the electric dipole operator of the radiation field. Initially, the eigenstates are occupied with Fermi distribution function $n_{F}\left(\epsilon_{\alpha^{\prime}}\right)$ about chemical potential $\mu$, and we insert the factor $\left[n_{F}\left(\epsilon_{\alpha^{\prime}}\right)-n_{F}\left(\epsilon_{\alpha}\right)\right]$ so that final states must be unoccupied. Then the additional current density due to the radiation is $\Delta J_{x}(\mathbf{R})=-e \int d \epsilon d^{2} \Delta r\left[n_{F}(\epsilon)-\right.$ $\left.n_{F}(\epsilon+\omega)\right] \sum_{\alpha^{\prime}}\left|\psi_{\alpha^{\prime}}\left(\mathbf{r}^{\prime}\right)\right|^{2} \delta\left(\epsilon-\epsilon_{\alpha^{\prime}}\right) w_{\alpha^{\prime}}(\mathbf{r}) \Delta x$. Here $\mathbf{R}=$ $\frac{1}{2}\left(\mathbf{r}+\mathbf{r}^{\prime}\right), \Delta \mathbf{r}=\mathbf{r}-\mathbf{r}^{\prime}=(\Delta x, \Delta y)$. For the average over disorder (denoted by an overbar throughout this paper), we write this expression as

$$
\begin{array}{r}
\Delta J_{x}(\mathbf{R})=-2 \pi e \int d \epsilon d^{2} \Delta r\left[n_{F}(\epsilon)-n_{F}(\epsilon+\omega)\right] \\
\times \mathcal{N}(\mathbf{r}, \epsilon+\omega) \mathcal{N}\left(\mathbf{r}^{\prime}, \epsilon\right) M\left(\mathbf{r}, \mathbf{r}^{\prime}\right) \Delta x
\end{array}
$$

where $\mathcal{N}(\mathbf{r}, \epsilon)=\overline{\sum_{\alpha}\left|\psi_{\alpha}(\mathbf{r})\right|^{2} \delta\left(\epsilon-\epsilon_{\alpha}\right)}$ is the average local density of states, which in high Landau levels obeys $\mathcal{N}\left(\epsilon+n \omega_{c}\right)=\mathcal{N}(\epsilon) . M\left(\mathbf{r}, \mathbf{r}^{\prime}\right)>0$ is defined by extracting these density of states factors, and is assumed to be independent of the energies $\epsilon, \epsilon+\omega$, and to be a function of $|\Delta \mathbf{r}|$ only. To measure the conductivity in linear response, a small dc electric field, $E_{d c}$, is applied in the $x$-direction. This tilts the energy spectrum as depicted in Fig. 11 and thus we assume that the only effect on Eq. (1) is to make $\mathcal{N}(\mathbf{r}, \epsilon)=\mathcal{N}\left(\epsilon-e E_{d c} x\right)$. $\Delta J_{x}$ vanishes to zeroth order in $E_{d c}$ by symmetry, and for linear response, we expand to first order in $E_{d c}$, and divide by $E_{d c}$ to find the longitudinal conductivity, $\sigma_{x x}$. We find that the radiation-induced change in the longitudinal conductivity is proportional to an integral of the partial derivative $\left(\partial\left(\mathcal{N}(\mathbf{r}, \epsilon+\omega) \mathcal{N}\left(\mathbf{r}^{\prime}, \epsilon\right)\right) / \partial \Delta x\right)_{\mathbf{R}}$. The density of states can be roughly modelled by $\mathcal{N}(\epsilon)=\mathcal{N}_{0}+\mathcal{N}_{1} \cos \left(2 \pi \epsilon / \omega_{c}\right)$. The final integral over $\epsilon$ can now be done, and at least for $\omega / \omega_{c}$ large compared to $\mathcal{N}_{0} / \mathcal{N}_{1}$, the result is

$$
\Delta \sigma_{x x} \propto-\sin \left(2 \pi \omega / \omega_{c}\right),
$$

with a positive coefficient. This form, which resembles the derivative of the density of states $\left.(\partial \mathcal{N} / \partial \epsilon)\right|_{\epsilon=\omega}$, arises because the main contribution is from initial states near the center of filled broadened Landau levels, which are scattered to empty broadened levels, and the available phase space is enhanced or diminished for $\Delta x$ positive or negative, depending on the energy change $\omega$ modulo $\omega_{c}$ (see Fig. (1). It is clear from experiment that $\sigma_{x y}$ is nearly 100 times larger than $\sigma_{x x}$ and is not significantly affected by the radiation. Therefore, inverting the conductivity tensor yields $\rho_{x x} \approx \rho_{x y}^{2} \sigma_{x x}$, which has the period and phase of the oscillations observed in experiment.

While the above treatment is highly oversimplified, it indicates that disorder plays a crucial role and may be all that is necessary to obtain the radiation-induced oscillations. This suggests that a diagrammatic (Kubo formula) calculation of the conductivity, including radia- tion and disorder but neglecting electron-electron interactions, will be sufficient to reproduce the effect. This calculation is presented below.

In the Landau gauge and the rotating-wave approximation (we neglect both counter-rotating and guiding-center terms in the coupling to radiation), the Hamiltonian is

$$
\begin{aligned}
H & =\sum_{k, n} n \omega_{c} c_{n k}^{\dagger} c_{n k}+\sum_{k, n ; k^{\prime}, n^{\prime}} c_{n k}^{\dagger} c_{n^{\prime} k^{\prime}} V_{n, k ; n^{\prime}, k^{\prime}} \\
& +\frac{e E \ell}{\sqrt{2}} \sum_{k, n} \sqrt{n}\left(c_{n k}^{\dagger} c_{n-1, k} e^{-i \omega t}+c_{n-1, k}^{\dagger} c_{n k} e^{i \omega t}\right)
\end{aligned}
$$

where $n$ is the Landau level index, $k$ is the $y$-component of momentum, $c_{n k}$ is the electron annihilation operator, $V_{n, k ; n^{\prime}, k^{\prime}}$ are the matrix elements of the disorder potential $V_{\mathrm{imp}}(\mathbf{r})$, and $E$ is the magnitude of the electric field component of the microwave radiation. Following the calculation of Ando [5] for the zero-radiation case, we shall include disorder within the self-consistent Born approximation (SCBA) and assume $\overline{V_{\mathrm{imp}}(\mathbf{r}) V_{\mathrm{imp}}\left(\mathbf{r}^{\prime}\right)}=$ $\left(2 \gamma / m^{*}\right) \delta\left(\mathbf{r}-\mathbf{r}^{\prime}\right)$ where $\gamma=1 / 2 \tau$ is the elastic scattering rate in zero magnetic field. This assumption of $\delta$-correlated disorder, while somewhat inappropriate for the long-ranged impurity potentials associated with modulation doping, significantly simplifies the problem by eliminating the momentum dependence of the self-energy. Yet, as we shall see, it manages to capture the important physics rather well. To all orders in the disorder and radiation, the Green's functions are given by the diagrams in Fig. 2(a). Since the radiation terms connect neighboring Landau levels, evaluating these diagrams for the retarded or advanced Green's functions, $G_{n m}^{R, A}\left(t_{1}, t_{2}\right)$, yields a matrix equation in Landau level indices $n$ and $m$. (The Green's functions are proportional to an identity matrix in the momentum label $k$, which is therefore dropped.) The presence of radiation renders this an inherently non-equilibrium problem which requires the approach of Kadanoff and Baym and Keldysh (see Refs. [6, 7, 8] for details). This involves the "lesser" Green's function, $G_{n m}^{<}\left(t_{1}, t_{2}\right)$, which is the only place where the distribution of electrons enters, and which is evaluated from the same set of diagrams via a slightly more complex procedure. It is convenient to define

$$
\bar{G}_{n m}^{R, A,<}(t, \mathcal{T})=e^{i n \omega t_{1}} G_{n m}^{R, A,<}\left(t_{1}, t_{2}\right) e^{-i m \omega t_{2}}
$$

where $t \equiv t_{1}-t_{2}, \mathcal{T} \equiv\left(t_{1}+t_{2}\right) / 2$, and $\bar{G}_{n m}^{R, A,<}(z, \mathcal{T})$, are the Fourier transforms in $t$. After some manipulation, we obtain the following system of equations:

$$
\begin{aligned}
\sum_{p} & {\left[\left(z-n\left(\omega_{c}-\omega\right)-\Sigma^{R, A}(z+n \omega) \pm i \delta\right) \delta_{n p}\right.} \\
& \left.-\frac{e E \ell}{\sqrt{2}}\left(\sqrt{n} \delta_{n, p+1}+\sqrt{p} \delta_{n+1, p}\right)\right] \bar{G}_{p m}^{R, A}(z)=\delta_{n m}
\end{aligned}
$$




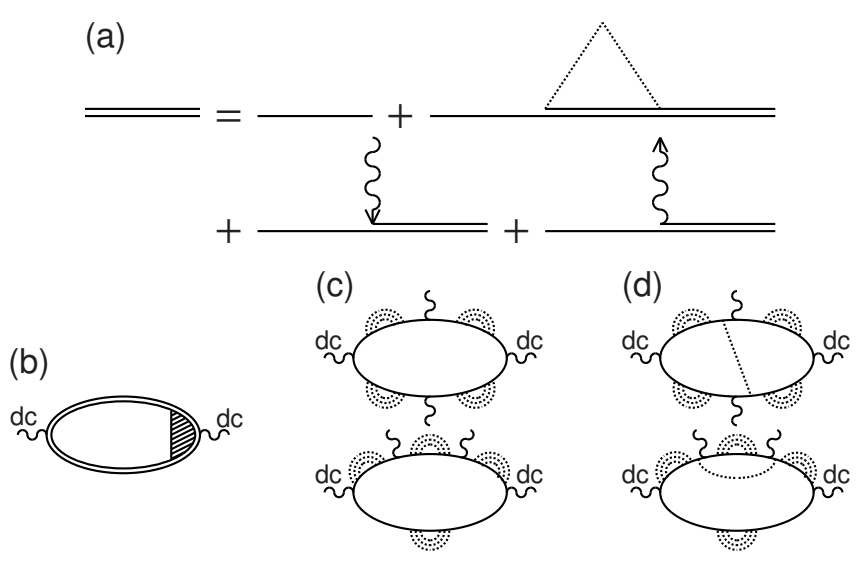

FIG. 2: Diagrams for (a) Green's function and (b) polarization bubble including radiation and disorder within SCBA. Disorder lines are dotted. Photon lines are curvy. The vertex of the polarization bubble is dressed with ladders of disorder lines. When the no-photons-crossed-by-disorder-lines conserving approximation is made, diagrams like those in (c) are included while those in (d) are neglected.

$$
\begin{aligned}
\bar{G}_{n m}^{<}(z)= & \sum_{p} \bar{G}_{n p}^{R}(z)\left[n_{F}(z+p \omega) 2 i \delta+\Sigma^{<}(z+p \omega)\right] \bar{G}_{p m}^{A}(z) \\
& \Sigma^{R, A,<}(z)=\frac{\gamma \omega_{c}}{\pi} \sum_{n} \bar{G}_{n n}^{R, A,<}(z-n \omega)
\end{aligned}
$$

where $\delta \rightarrow 0^{+}$. The Eqs. (5 7) have been displayed for the steady state case, in which all Green's functions are independent of the average time, $\mathcal{T}$. The general $\mathcal{T}$ dependent situation leads to more complicated expressions which can be obtained as discussed in Ref. 6. Given the Green's functions, the conductivity is obtained via the Kubo formula, $\sigma_{i j}=-\lim _{\Omega \rightarrow 0} \operatorname{Im} \Pi_{i j}^{R}(\Omega) / \Omega$, where $\Pi_{i j}^{R}$ is the retarded current-current correlation function, and $i, j=\{x, y\}$. Diagrammatically, this means evaluating the polarization bubble in Fig. 2(b) where the vertex is dressed by ladders of impurity lines.

An exact solution is complicated by the self-consistent nature of the above equations. We shall simplify by neglecting vertex corrections to the polarization bubble. To do so within a conserving approximation, we must also neglect all diagrams in which impurity lines cross photon insertions (see Fig. 22(c,d)). Note that this is equivalent to replacing the fully self-consistent $\Sigma$ 's in the above equations with $\Sigma$ 's calculated in the absence of radiation but still self-consistent in the disorder. Thus, if we define $\Sigma(z)$ to be the self-energy calculated by Ando [5] for the zero-radiation case, then $\Sigma^{R}(z) \rightarrow \Sigma(z), \Sigma^{A}(z) \rightarrow \Sigma^{*}(z)$, and $\Sigma^{<}(z) \rightarrow-2 i n_{F}(z) \operatorname{Im} \Sigma(z)$. This approximation also has the virtue of mimicking energy loss mechanisms so that a steady state $\mathcal{T}$-independent solution does indeed exist 9], and can be obtained directly from Eqs. (516). The radiation enters only when the Green's functions are calculated from the $\Sigma$ 's. In the absence of vertex correc- tions, it is straightforward to calculate the conductivity from the Green's functions. We find that

$$
\begin{aligned}
\left\{\begin{array}{l}
\sigma_{x x} \\
\sigma_{x y}
\end{array}\right\}= & -\frac{e^{2} \omega_{c}^{2}}{4 \pi^{2}} \sum_{n, m} \sqrt{(n+1)(m+1)} \int d z \\
& \times\left[\operatorname{Im} \bar{G}_{n+1, m+1}^{<}(z) \frac{d}{d z}\left\{\begin{array}{l}
\operatorname{Im} \\
\operatorname{Re}
\end{array}\right\} \bar{G}_{n m}^{R}(z+\omega)\right. \\
& \left. \pm \operatorname{Im} \bar{G}_{n m}^{<}(z) \frac{d}{d z}\left\{\begin{array}{l}
\operatorname{Im} \\
\operatorname{Re}
\end{array}\right\} \bar{G}_{n+1, m+1}^{R}(z-\omega)\right]
\end{aligned}
$$

and obtain the resistivity by inverting the $\sigma$-matrix.

Within the approximations discussed above, and using parameter values appropriate to the experiments of Ref. 2, we have calculated $\rho_{x x}$ numerically to all orders in radiation and disorder. Results are presented in Fig. 3 . where we plot $\rho_{x x}$ versus $1 / \omega_{c}$ for fixed $\omega$. We consider three values of radiation intensity (power per unit area in units of $m^{*} \omega^{3}$ ): $I=0$ (dark), $I=0.0034$ (moderate intensity), and $I=0.0115$ (high intensity). The dark resistivity exhibits only the familiar $\mathrm{SdH}$ oscillations which have period $1 / \mu$ and decay away as $\omega_{c}$ becomes small compared with the temperature. For moderate intensity, we find a pronounced radiation-induced oscillation of $\rho_{x x}$. In agreement with experiment, the period of oscillation is $1 / \omega$ and minima are found near $\omega / \omega_{c}=$ integer $+1 / 4$. We note, however, that the $1 / 4$ phase shift is not universal, varying between 0 and $1 / 2$ depending upon disorder and intensity. A more robust feature is the presence of zeros of the oscillation at the integer values of $\omega / \omega_{c}$. Unlike the $\mathrm{SdH}$ oscillations, the radiation-induced oscillations are not sensitive to the temperature-broadening of the electron distribution about the Fermi level. $T$-dependence therefore derives from the the $T$-dependence of the scattering, a feature absent from the present calculation due to our neglect of interaction effects. The magnitude of the radiation-induced oscillations can easily exceed the dark resistivity, leading to regions of negative total resistivity. This is reasonable for a non-equilibrium system, and a similar effect has been observed experimentally in semiconductor superlattices [10], but it seems that some additional physics is required for these negative-resistivity minima to become the zero-resistance states observed in the present experiments. For high intensity radiation, additional features appear which have yet to be discovered experimentally. These likely correspond to multi-photon processes by which an electron absorbs $m$ photons and is promoted by $n$ Landau levels.

Since the simplifying approximations discussed above, employed to make the calculation tractable, are not well controlled, we cannot expect our results to be quantitatively accurate. However, the fact that even this simplified calculation captures the qualitative form of the experimental results encourages us that we have identified the essential physics of the problem. Due to the assumption of $\delta$-correlated disorder, even the zero-radiation (dark) resistivity, $\rho_{x x}^{0}$, does not agree quantitatively with 

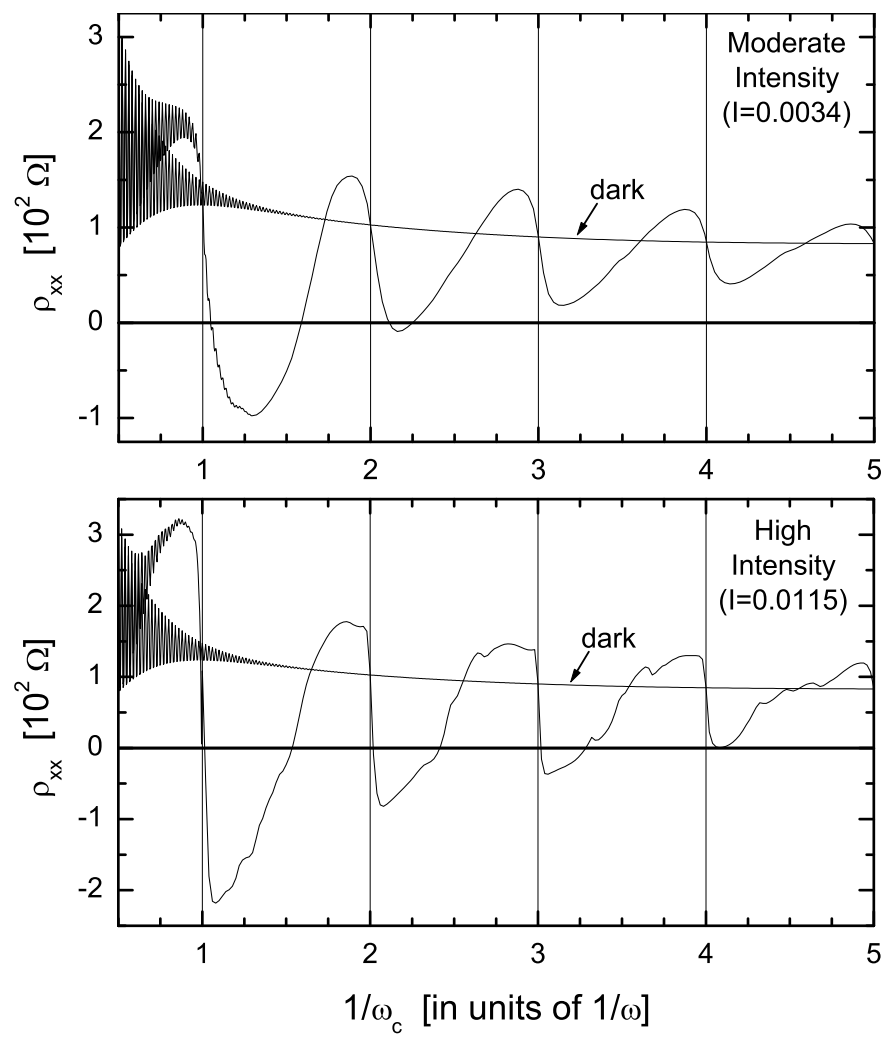

FIG. 3: Calculated radiation-induced resistivity oscillations. We plot $\rho_{x x}$ vs $1 / \omega_{c}$ at fixed $\omega$ for $\mu=50 \omega, k_{B} T=\omega / 4$, $\gamma=0.08 \omega$, and three values of radiation intensity (in units of $\left.m^{*} \omega^{3}\right): I=0$ (dark), $I=0.0034$ (upper panel), and $I=$ 0.0115 (lower panel). For computational purposes, the energy spectrum is cutoff at 20 Landau levels above and below the chemical potential. The high-frequency oscillations seen at small $1 / \omega_{c}$ are the familiar $\mathrm{SdH}$ oscillations with period $1 / \mu$.

experiment. As the calculation neglects the difference between the transport lifetime, $\tau_{t r}$, and the single particle lifetime, $\tau$, it overestimates $\rho_{x x}^{0}$ by a factor of $\tau_{t r} / \tau$, equal to about 50 in the present case. Since the dark Hall resistivity, $\rho_{x y}^{0}$, is approximately independent of disorder in the $B$-field regime of interest, the calculated value of $\rho_{x y}^{0}$ is correct, but effectively too small compared to $\rho_{x x}^{0}$. This quantitative error in the dark resistivity has two important consequences. When we calculate the radiationinduced change in the Hall resistivity, $\Delta \rho_{x y}$, we find that it is also oscillatory and of comparable magnitude to $\Delta \rho_{x x}$. Since $\rho_{x y}^{0}$ is effectively too small, we find a total Hall resistivity that exhibits noticeable radiation-induced oscillations. For analogous reasons, while our calculations yield oscillations in $\sigma_{x x}$ which grow linearly with radiation intensity, the intensity-dependence is muted when we invert the conductivity matrix to obtain $\rho_{x x}$. However, correcting for the missing factor of 50 , it is clear that the effect of radiation on $\rho_{x y}$ is negligible and that the oscillations in $\rho_{x x}$ grow linearly with intensity, which is what was observed experimentally.
In conclusion, we understand the radiation-induced magnetoresistance oscillations recently observed in the 2DEG to be a consequence of photo-excited disorderscattered electrons contributing to the dc resistivity in an oscillatory manner inherited from the broadened Landau level structure of the energy spectrum. Our diagrammatic calculation yields a radiation-induced oscillation of $\rho_{x x}$ that has the correct period and phase. We find that for radiation of sufficient magnitude, the amplitude of the oscillation can be made large enough that the total resistivity is negative in the vicinity of the minima. This effect is reminiscent of the experimentally observed zero-resistance states but indicates that additional physics, perhaps localization or electron-electron interactions, is required to understand this aspect of the observed phenomenon. Our calculations at high intensity reveal multi-photon effects, the observation of which should be a goal for future experiments. More advanced calculations, going beyond the simplifying approximations employed herein and including the interaction effects responsible for energy relaxation and temperature dependence, are left for future work.

We are grateful to K. Lehnert who brought to our attention the experiments of Ref. 10, which helped inspire the physical picture discussed in this Letter. We are also grateful to R. Willett for raising the possibility of negative resistance in these systems. We thank R. G. Mani, R. Shankar, and R. Willett for helpful discussions. This work was supported by NSF Grants DMR-0103639 (A.C.D.), DMR-0098226 (S.S. and A.C.D.), and DMR0196503 (S.M.G. and A.C.D.).

After submitting this Letter for publication, we learned that a related physical picture and a calculation of the conductivity to second order in the radiation, with a prediction of negative conductivity, were previously reported by Ryzhii et al. [11, 12].

[1] M. A. Zudov et al., Phys. Rev. B 64, 201311 (2001)

[2] R. G. Mani et al., Nature 420, 646 (2002)

[3] M. A. Zudov et al., Phys. Rev. Lett. 90, 46807 (2003)

[4] W. Kohn, Phys. Rev. 123, 1242 (1961)

[5] T. Ando, J. Phys. Soc. Japan 37, 1233 (1974)

[6] H. Haug and A. Jauho, Quantum Kinetics in Transport and Optics of Semiconductors (Springer, Berlin, 1996)

[7] L. P. Kadanoff and G. Baym, Quantum Statistical Mechanics (Benjamin/Cummings, Massachusetts, 1962)

[8] J. Rammer and H. Smith, Rev. Mod. Phys. 58, 323 (1986)

[9] In the quantum Boltzmann equation, this approximation corresponds to having a given state repopulated by an "in" scattering rate from states at other momenta and Landau levels with a radiation-independent thermal occupation.

[10] B. J. Keay et al., Phys. Rev. Lett. 75, 4102 (1995)

[11] V. I. Ryzhii, Fiz. Tverd. Tela 11, 2577 (1969) [Sov. Phys. 
Solid State 11, 2078 (1970)]

[12] V. I. Ryzhii et al., Fiz. Tekh. Poluprovodn. 20, 2078
(1986) [Sov. Phys. Semicond. 20, 1299 (1986)] 